\title{
Kernos
}

Revue internationale et pluridisciplinaire de religion grecque antique

$13 \mid 2000$

Varia

\section{Verso una tipologia delle religioni classiche}

\section{Fabio Mora}

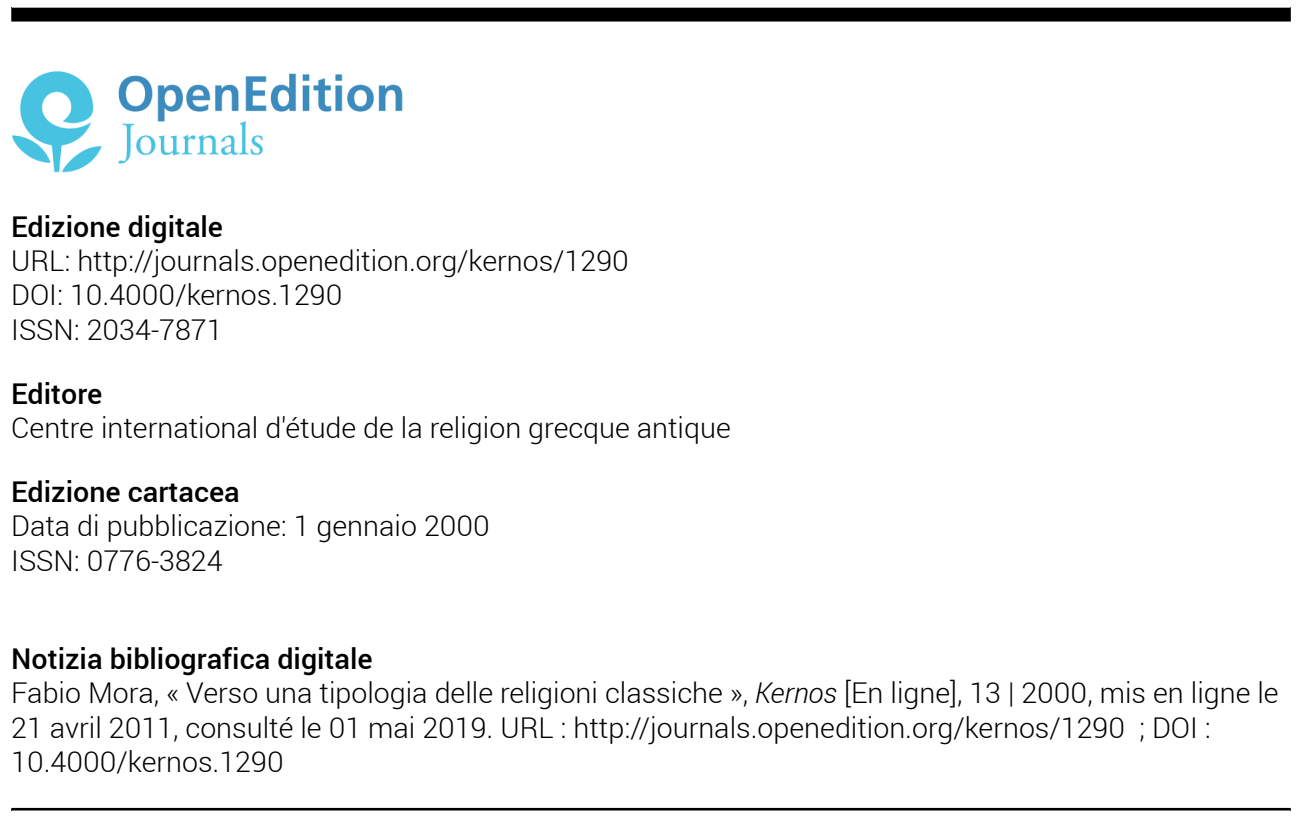

Kernos 
Kernos, 13 (2000), p. 9-33.

\section{Verso una tipologia delle religioni classiche}

\section{La Storia delle Religioni: oggetto e metodo}

Una lunga discussione ha animato gli studi di Storia delle Religioni, e cioè la questione della definibilità dell'oggetto della materia, quale necessaria premessa scientifica dell'indagine storico-religiosa: la questione tocca due aspetti tra loro complementari, e cioè i temi dell'universalità e della specificità dell'esperienza religiosa. Si tratta cioè di stabilire da un lato se sia possibile definire un modello unico, generalissimo di religione per procedere allo studio universale della Religione, dall'altro se si possa individuare una struttura fondamentale del comportamento religioso, come ambito più specifico del comportamento umano, individuale e collettivo.

Una buona risposta alla prima questione è stata data da U. Bianchi ${ }^{1}$, sostituendo all'alternativa secca sì/no la distinzione tra interpretazioni univoche, equivoche ed analoghe dei fatti religiosi: ponendo cioè una terza prospettiva tra la tesi dei sostenitori di un'unica struttura religiosa dell'uomo di ogni tempo ed epoca, in particolare quale rapporto con il sacro ${ }^{2}$ (un concetto che può essere giustamente considerato una trasposizione attenuata dell'oggettiva esistenza di Dio - quindi un presupposto piuttosto teolo-

1 Cf. U. BIANchi, Saggi di metodologia della Storia delle Religioni, Roma, 1979, partic. p. 59, 65-95, 111 sq. La problematica introdotta dal Wittgenstein (per cui v. n. 9) è stata recentemente rivalutata su un piano diverso, più operativo, ma del tutto compatibile col concetto di analogia, suggerendo l'uso dei concetti di fuzzy sets e polythetic classes: $c f$. J.A.M. SNOEK, Classification and Definition Theory, an Overview, in U. BIANCHI (ed.), The Notion of "Religion" in Comparative Research, Selected Proceedings of the XVItb Congress of the International Association for the History of Religions, Rome, 3rd-8th September, 1990 (in seguito: Notion), Roma, 1994, p. 741-754.

2 ì la posizione ancora recentemente sostenuta da J. RIEs, L'uomo religioso e il sacro alla luce del nuovo spirito antropologico, in J. Ries (ed.), Trattato di antropologia del sacro (in seguito: Trattato), I. Le origini e il problema dell'bomo religiosus, Milano, 1989, p. 3558 , sulla base di una lunga tradizione che parte da E. Dur кherm (Les formes élémentaires de la vie religieuse, Paris, 1912) e R. Oтто (Das Heilige, Gotha, 1917) e passa attraverso la fenomenologia religiosa di G. VAN DER LEEUW (Pbänomenologie der Religion, Tübingen, $1956^{2}$ ) ed anche la tendenza fenomenologica del primo Eliade, nel Traité d'bistoire des religions, Paris 1949 in parte attenuatasi nella sua ultima sintesi, Histoire des croyances et des idées religieuses, I-III, Paris, 1976-1983, tr. it. Firenze, 1979-1983. 
gico che scientifico dell'indagine ${ }^{3}$ ) e l'opposta idea della dissoluzione dell'oggetto storicoreligioso ${ }^{4}$, con il passaggio dalla Storia delle Religioni all'indagine antropologica di singole civiltà; tale prospettiva presuppone significative continuità parziali tra singole religioni, che permettono (nel caso limite) di riscontrare una sostanziale affinità anche tra religioni non direttamente confrontabili, ma che risultino a loro volta comparabili con un terzo elemento ${ }^{5}$. Questo concetto di analogia riporta opportunamente la discussione metodologica da un piano astrattamente teologico-filosofico ${ }^{6}$ ad uno concretamente storico: la sistemazione concettuale ${ }^{7}$ dei fatti empiricamente esperiti come religiosi può avvenire soltanto a posteriori come frutto della conoscenza storica e non deve precedere, pregiudizialmente, come schema a priori l'indagine storico-religiosa ${ }^{8}$.

La scelta di basare il concetto di analogia non sulla distinzione aristotelica di genere-specie, ma sulla 'somiglianza familiare' introdotta dal Wittgenstein $^{9}$ e dotata di ben maggiore pervasività ed astrazione implica d'altronde la rinuncia ad un modello generalissimo dei fatti religiosi. In questo modo si risponde anche alla seconda questione, quella dei confini ${ }^{10}$ tra fatti religiosi $e$ non religiosi e quindi dell'oggetto degli studi di Storia delle Religioni: l'oggetto di questa disciplina non sarà un insieme obbiettivamente ed universalmente definito di fenomeni, ma in via primaria l'insieme dei fenomeni soggettivamente esperiti come religiosi ${ }^{11}$, ed in via secondaria quei fenomeni che, per

3 E la critica giustamente formulata da B. GLAdigow, Gegenstände und wissenschaftlicher Kontext von Religionswissenschaft, in H. CANCIK, B. Gladigow, M. LAUBSCHER (eds), Handbuch religionswissenschaftlicher Grundbegriffe, I, Stuttgart 1988, p. 26-38 partic. p. $29 s q$.

4 È la posizione ad es. di D. SabBatucci, Kultur und Religion, ibid. p. 43-58.

5 Bianchi, Saggi, cit. (n, 1), p. $58 \mathrm{sq}$.

6 Anche di una teologia rovesciata, quale quella che sorregge molte forme riduzionistiche di interpretazione dei fatti religiosi come epifenomeno: Branchi, Saggi, cit. (n. 1), p. 90 .

7 Molto utili sul piano metodologico appaiono le rifléssioni sul processo di classificazione svolte da C. CoLPE, Religionstypen und Religionsklassen. Von der Verwechslung zur Unterscbeidung, in Notion, cit. (n. 1), p. 645-660.

8 Bianchi, Saggi, cit. (n. 1), p. 143. Cf. anche G. SFAMENI GASPARro, History of Religions: A Retrospective and Prospective View, in Notion, cit. (n. 1), p. 913-917; BrancHI, Concluding Remarks. The History of Religions, Today, ibid., p. 919-921.

9 Analizzando il concetto di gioco: L. Witrgenstein, Pbilosopbiscbe Untersucbungen, Oxford, 1953, $8 \int 65-77$.

10 Per la convenzionalità dei confini quali strumenti ad boc cf. ibid. $\$ 69$.

11 Giustamente Gladigow, art. cit. (n, 3), p. 38 rifiuta la pretesa di Smith che ogni giudizio storico-religioso debba rispecchiare la soggettiva autocomprensione del gruppo oggetto di indagine, per sottolineare la necessità di descrivere i fatti religiosi con un metalinguaggio distinto dal linguaggio religioso interno a singole religioni; sui problemi connessi con la creazione di un simile metalinguaggio (anche come sistema articolato di concetti) a partire dal linguaggio concreto della tradizione religiosa, prevalentemente eu- 
la loro analogia con quelli percepiti come religiosi in altre civiltà, possono essere ugualmente considerati religiosi, pur non essendo soggettivamente esperiti come tali, ed in particolare tra essi i residui e le forme attenuate di religiosità.

Per questa via si arriva a stabilire il procedimento operativo della Storia delle Religioni, quale produzione di una complessa tipologia storica delle religioni e dei motivi religiosi - orientata, nel primo caso, verso il principium individuationis di una singola civiltà od ambiente più specifico, nel secondo verso il grado di generalità (piuttosto che universalità) di determinati ' ingredienti ' del fenomeno religioso. Quale definizione intuitiva di religione e fattore di orientamento nell'analisi dei fenomeni potenzialmente religiosi varrà poi l'idea che la religione rappresenti, produca, costituisca una rottura di livello ${ }^{12}$, cioè l'introduzione di un elemento ' altro ' dalla realtà esperita ${ }^{13}$ quale elemento essenziale dell'esperienza. Di questa definizione deve essere sottolineato in primo luogo il carattere funzionalistico, che non individua quindi una forma generalissima, ma soltanto un'analogia di funzione rispetto all'insieme dei dati antropologici, al cui interno possono darsi elementi di discontinuità più o meno forti, ma mai totale divergenza. La polarità tra dati antropologici empiricamente riscontrabili, l'elemento o gli elementi cui è affidata la rottura di livello e l'insieme rappresentato da ogni singola cultura rende d'altronde ragione della difficoltà di ridurre ad un minimo comun denominatore il concetto di religione, a torto addotta come prova del suo carattere equivoco: il grado in cui si compie la rottura di livello potrà risultare volta a volta diverso, così come la materia impiegata e la forma (intesa quale maggiore o minore distinzione di sfere separate). Se la religione si caratterizza come rottura di livello rispetto alla totalità di una cultura (o sub-cultura) e questa rottura di livello è caratterizzata da diversi gradi di libertà, la ricerca di modelli comuni, per quanto generalissimi ed estremamente formali, non potrà arrivare oltre il livello penultimo delle singole opzioni attivabili per operare questa rottura di livello. Sarebbe però sbagliato ridurre la profonda analogia dei fatti religiosi, quale risulta empiricamente accertabile, ad una federazione di prodotti univoci al loro interno, ma tra loro equivoci: lo studio del

ropea in cui è sorta la Storia delle Religioni $c f$. B. SALER, Cultural Antbropology and the Definition of Religion, in Notion, cit. (n. 1), p. 831-836.

12 Sul concetto di rottura di livello, introdotto da Eliade, $c f$. U. Bianchi, La Storia delle Religioni, in G. Castellani (ed.), Storia delle religioni, Torino, $1970^{6}$ (in seguito: Storia delle religioni), I, p. 1-168, partic. p. 160 . Una formulazione diversa, ma altrettanto adeguata viene data, in termini forse più chiari e moderni, da J.G. PLAtvoet, Defining the Definers: Non-Verifiability/Non-Falsifiability as a Definiens in an Operational Definition of Religion, in Notion, cit. (n. 1), p. 701-712, partic. p. 711 che identifica nella non-falsificabilità/non-verificabilità (in senso popperiano) il proprium degli elementi dell'esperienza religiosa.

13 Od anche, nella sua forma più attenuata: di una diversa percezione della realtà esperibile. 
difficile rapporto reciproco di religioni radicalmente diverse conviventi nella stessa cultura dimostra significativamente come la presenza di opzioni comuni ed altre diverse, di una direzione diversa, ma di una forma o materia comuni, nella rottura di livello simultaneamente operata renda assai difficile non solo la comprensione dell'altro, ma la stessa propria autocomprensione religiosa; ciò che evidentemente non si verificherebbe se l'esperienza religiosa fosse rigorosamente segmentabili in diversi comportamenti stagni, anziché costituire un continuum polarizzato - in maniera non sempre adeguata intorno ad alcune opzioni fondamentali.

La rappresentazione dell'esperienza religiosa come un continuum polarizzato permette d'altronde di impostare in maniera nuova la questione dei confini tra la Storia delle Religioni e le discipline affini: un problema che si pone in maniera più marcata per questa che non per altre discipline, in ragione della più marcata interdisciplinarietà, che deriva dal carattere prevalentemente funzionale del concetto di ' rottura di livello '. L'estrema varietà di materia, forma, metodo di tale 'rottura di livello' porta abbastanza naturalmente a casi di doppia appartenenza: a fenomeni cioè che risultano rilevanti per la Storia delle Religioni per la rottura di livello che operano, ma al tempo stesso rientrano in altre discipline, per la forma specifica di attività umana che presuppongono; sorgono così aree di confine, più frequenti quando si tratta di attività intellettuale individuale, ma numerose anche nel caso dei fenomeni collettivi. Una delimitazione della disciplina, in senso letterale, operata cioè tracciando dei confini rigidi, risulta esiziale per la Storia delle Religioni, in quanto suggerisce la rinuncia ad occuparsi a pieno titolo di tali aree di confine. Sarà invece opportuno definire la Storia delle Religioni in rapporto al luogo centrale del suo interesse, e cioè la rottura di livello diversamente presente in ogni singola civiltà, ma considerarla priva di confini precisi, perché strutturalmente aperta ad una interdisciplinarietà a tutto campo: vi sono momenti della storia religiosa (in primis il neoplatonismo) in cui il luogo centrale della ricerca storico-religiosa non è costituito dall'elemento tradizionalmente religioso, ma da un elemento già oggetto di altra ' più specifica ' disciplina ${ }^{14}$.

Si potrà poi porre la questione del confine tracciabile tra fenomeni religiosi e parareligiosi, per quanto riguarda la loro suscettibilità o meno a rappresentare l'elemento di comparazione utile a definire il carattere religioso di un altro fenomeno solo parzialmente analogo: saremmo propensi a riconoscere questo carattere religioso a quegli elementi che comunque compaiono nell'elemento centrale dell'esperienza religiosa; quindi anche alle speculazioni filosofico-religiose del neoplatonismo o delle filosofie indiane.

14 Nella stessa prospettiva si può affrontare l'annosa questione di determinati fenomeni parareligiosi, come ad es. la magia: l'alterità della magia rispetto alla religione andrà colta in una più ridotta analogia tra magia e religione, probabilmente a livello delle opzioni fondamentali; ma ciò non esclude un'indagine storico-religiosa della magia. 


\section{La tipologia storica delle religioni ed il rapporto con le fonti}

Fondamentale nella prospettiva metodologica del Bianchi - in ragione della rinuncia ad una definizione preliminare di religione e della tensione ad una sempre maggiore approssimazione del concetto di religione attraverso la ricerca storica - è il ruolo di una tipologia storica, che accerti elementi di continuità od anche di contrasto all'interno di un ambito culturale ben più vasto della singola civiltà, ma ancora relativamente omogeneo: così da evitare astratte comparazioni puramente fenomenologiche, tra usi e pratiche staccati dal proprio contesto e quindi usualmente fraintesi ed arbitrariamente identificati o contrapposti ${ }^{15}$, in un processo che riposa in larga misura su una implicita ontologia della religione ${ }^{16}$, quale sistematica organizzazione dell'esperienza religiosa, che dell'aprioristica definizione preliminare della religione ${ }^{17}$ sviluppa le premesse o l'intuizione originaria.

Il concetto di tipologia storica ${ }^{18}$ è d'altronde l'unico in grado di rispondere in maniera adeguata all'evidente esplosione quantitativa dell'oggetto degli studi storico-religiosi ed evitare che l'impossibilità pratica di dominare l'insieme delle religioni porti alla dissoluzione dell'oggetto storico-religioso, quale prodotto di un'indagine non esclusivamente idiografica, ma anche ed in primo luogo comparativa. Un ragionevole equilibrio tra l'ambito ristretto delle competenze individuali, di attiva ricerca storico-filologica, del singolo studioso e l'irrinunciabile esigenza che ogni indagine storico-religiosa resti aperta e rifluisca nella più ampia comparazione storico-religiosa ${ }^{19}$, volta all'elaborazione, graduale e paziente, di un concetto storico di religione, può essere a nostro avviso ottenuto con la distinzione, in linea di massima, di due diversi livelli di comparazione: una comparazione di primo livello tra concreti fatti storico-religiosi tra loro prossimi, volta ad inserirli in un'articolata tipologia storica, ed una comparazione di secondo livello, tra i diversi, complessi tipi storici così individuati, per risalire da forme geograficamente e cronologicamente delimitate di religione ad un più generale concetto, storicamente acquisito, di religione.

Se la comparazione storico-religiosa risulta necessaria per la valutazione del carattere religioso dei fatti documentati, la loro interpretazione e l'individuazione di tipologie storiche e solo al metodo storico-comparativo spetta la ricostruzione complessiva del singolo fenomeno e la sua classificazione in relazione ai fenomeni comparabili, la ricerca deve però nutrirsi di continua attenzione al carattere storico dei fatti studiati, mediante il loro accertamento puntuale, idiografico. Sotto questo aspetto la storia delle religioni riposa

\footnotetext{
Bianchi, Saggi, cit. (n. 1), p. 20-23.

Bianchi, Saggi, cit. (n. 1), p. 83.

17 V. oltre infra n. 27.

18 BIANCHI, Saggi, cit. (n. 1), p. 56 sq., 145 sq.

19 BIANCHI, Saggi, cit. (n. 1), p. 20 sq.
} 
prevalentemente sul più generale metodo storico (o, dove sia prevalente l'uso di fonti scritte, storico-filologico): prevalentemente, in quanto spetta alla sensibilità dello storico delle religioni porre domande motivate dalle conoscenze storico-comparative, ma l'accertamento delle risposte dipende dal corretto uso delle metodologie storiche necessarie a verificare fatti specifici nelle civiltà in questione.

Il metodo storico si pone come ausiliario del metodo storico-comparativo, quale strumento che lo storico delle religioni deve dimostrare di usare correttamente in ogni sua concreta verifica. Su questo piano più propriamente storico acquista una particolare rilevanza la questione del genere di fonti disponibili per i diversi ambiti culturali, che condiziona significativamente le possibilità ed i metodi d'indagine dello storico, dello storico della civiltà e quindi anche più puntualmente dello storico delle religioni.

In una rapida, forse un po' sommaria tipologia ${ }^{20}$, si possono utilmente distinguere, dal punto di vista dell'indagine cui possono essere sottoposti, $\mathrm{i}$ seguenti tipi di civiltà:

a) civiltà contemporanee, la propria od ' altra ', interrogabili a piacere e in ragione della propria competenza ( $v$. sociologia ed etnologia religiosa), in cui lo stesso storico opera come fonte secondaria prossima ai fatti;

b) civiltà moderne, in possesso della scrittura, della stampa e di archivi, che presentano sovrabbondanza di fonti dirette, non particolarmente puntuali, difficilmente padroneggiabili nel loro insieme (p. es. atti di processi alle streghe, letteratura devozionale, relazioni episcopali, ecc.), coniugate a fonti secondarie prossime ai fatti;

c) civiltà dotate di scrittura, di fonti monumentali e scritte, di vario carattere comprendenti fonti secondarie (testimonianze riflesse), non sempre abbondanti, talvolta lacunose;

d) civiltà dotate di scrittura e di fonti scritte dirette abbondanti ma ristrette ad alcuni generi letterari, eventualmente implicanti particolari problemi di pubblicazione e/o traduzione e/o di datazione con riferimento al loro carattere di testimonianza religiosa e generalmente prive di fonti secondarie prossime ai fatti e prodotte da appartenenti a quella stessa civiltà;

e) civiltà non dotate di scrittura, note attraverso fonti monumentali, tradizioni orali e fonti secondarie prodotte da membri di una civiltà terza rispetto a quella oggetto e a quella moderna, soggetto di indagine storica;

f) civiltà non dotate di scrittura, note solo attraverso fonti monumentali e/o tradizioni orali.

Sul piano storico ottimale è la situazione sub a), in cui la ricerca dipende solo dalla capacità dello storico di formulare questioni pertinenti e di applicare i metodi propri delle scienze sociali od etnologiche: però le possibilità di comparazione, necessaria per il costituirsi di un giudizio storico-religioso, per

20 È evidente che in taluni casi la presenza di parte dei tratti rilevanti genera situazioni intermedie che qui sarebbe pedante elencare minutamente. 
l'individuazione cioè di un adeguato contesto interpretativo, appaiono ridotte, in ragione della relativa omogeneità degli ambiti studiati. La situazione sub b) appare sotto questo aspetto già preferibile, in quanto ammette anche un'indagine diacronica, di fatto però prevalentemente all'interno od in relazione con una sola civiltà, quella occidentale, ciò che riduce di molto lo spettro dei fenomeni osservabili.

La maggior parte delle civiltà che lo storico delle religioni deve conoscere, comprendere e comparare, secondo le specifiche esigenze di ogni ricerca tematica, appartiene però alle ultime quattro categorie citate, che abbiamo ritenuto di distinguere sulla base del possesso o meno della scrittura ${ }^{21}$ e della qualità delle fonti secondarie prossime ai fatti. Nel caso dei popoli dotati di scrittura, su cui si danno in genere fonti secondarie, a volte ampie a volte povere, la distinzione principale ci sembra passare tra quelle civiltà le cui fonti secondarie sono testimonianze riflesse prodotte da individui di quella civiltà e civiltà per cui abbiamo solo o prevalentemente fonti secondarie prodotte da una cultura terza alla civiltà-oggetto ed alla nostra civiltà. Nel caso dei popoli privi di scrittura - nella misura in cui non sono più osservabili e non rientrano quindi più nella categoria sub a), ad es. perché ormai in una fase di rapida acculturazione - la distinzione maggiore ci appare quella dell'esistenza o meno di (significative) fonti secondarie prossime ai fatti: in loro assenza il metodo storico diventa più propriamente paletnologico e/o folkloristico (con tutti i problemi che pone la datazione dei vari strati della tradizione orale e dell'individuazione dei frequenti influssi). La categoria sub c) è probabilmente quella in cui meglio si possono classificare le c.d. grandi religioni, che però - in parte, per i loro sviluppi contemporanei - partecipano della categoria b). Se sotto questo riguardo la nostra classificazione può risultare insufficiente (in quanto le grandi religioni sorgono in contesti documentari molto diversi da quelli in cui oggi si trovano ad operare, passano quindi di fatto da una categoria all'altra), essa ci permette di individuare in modo particolare tramite le quattro categorie $\mathrm{da}$ c) ad f) i diversi problemi di indagine storica posti dalle religioni del mondo classico e dei popoli limitrofi.

\section{Le religioni classiche nell'ambito della Storia delle Religioni}

'Mondo classico ' è termine che si presta a diverse significazioni: la definizione più riduttiva limita il concetto agli ambiti in cui sono parlate le lingue classiche (greco e latino); noi riteniamo più opportuno, proprio per l'importanza delle fonti secondarie greche e latine per la conoscenza di altre alte culture dotate di scrittura, comprendervi tutte le civiltà dotate di scrittura note agli autori greci e latini, in aree poi oggetto di penetrazione greca o

21 O meglio: di un suo consistente utilizzo in condizioni di intellegibilità da parte dei moderni, in quanto i relitti di molte lingue antiche semi-sconosciute - ad es. messapico, retico, leponzio - risultano dal nostro punto di vista pressoché irrilevanti. 
latina ${ }^{22}$, da Omero all'espansione $\operatorname{araba}^{23}$ : non solo Greci e Latini, ma anche Etruschi, Italici, Egizi, Fenici, Ebrei ${ }^{24}$, altri popoli semitici, civiltà mesopotamiche, micrasiatiche, Iran in una certa fase della loro storia appartengono a nostro avviso a pieno titolo alla koine culturale ellenistico-romana. Dal punto di vista storico-religioso, per rispettare gli elementi di continuità interni alle civiltà dotate di scrittura più antiche del mondo classico o periferiche rispetto al mondo greco-romano, si è però ovviamente portati a coniugare col concetto di 'Mondo classico ' l'altro di 'Alte culture del mondo antico', che include le civiltà già ricordate, dalla scoperta della scrittura alla fine del mondo antico. Ma le culture superiori, dotate di scrittura, del mondo classico vivono in stretto contatto con popolazioni prive di scrittura, cui sono anche in buona misura linguisticamente imparentate (Celti, Germani, Sciti, popolazioni ' mediterranee '): di qui l'esigenza di combinare lo studio delle Religioni del mondo classico con quelle dei 'Popoli limitrofi', anche in ragione dell'uso delle stesse fonti secondarie per entrambe. L'uso delle stesse fonti non deve però far trascurare un ultimo confine, che separa l'area compresa nella definizione più ristretta di mondo classico da quella da noi aggiunta per parlare di Mondo classico e popoli limitrofi, in ragione della diversa relazione tra le fonti secondarie e i fatti descritti: infatti le fonti greco-latine sono prevalentemente testimonianze riflesse sulla propria civiltà e religione, testimonianze esterne sulle alte culture non greco-latine e sulle civiltà orali dei popoli limitrofi od infine testimonianze incrociate, interne al mondo classico in senso ristretto, ma 'non riflesse' di autori greci che scrivono di cose romane o autori latini che si occupano di cose greche.

Una riflessione sulla natura delle fonti disponibili ci sembra importante, perché la forte specificità, il carattere particolare delle notizie ha sempre influenzato grandemente la natura della ricerca e della ricostruzione della

22 È cos̀i possibile porre un limite ragionevole al continuum che lega le alte culture del mondo classico ad altre culture di cui greci e latini ebbero una qualche conoscenza, sulla base della rilevante correlazione tra effettiva penetrazione e valore delle notizie riferite: il limite orientale della civiltà classica va quindi individuato nel regno di Bactriana, tra Iran orientale ed India occidentale.

23 Rispetto ai classici limiti temporali della civiltà classica (fine dell'Impero Romano d'Occidente nel 476, morte di Giustiniano) questa datazione bassa, originariamente proposta dal Pirenne, corrisponde ad una radicale cesura nella storia culturale e religiosa di lungo periodo; la datazione alta, di scuola anglosassone, che esclude il periodo dell'Impero cristiano ( $m a$ anche del tardo paganesimo!), appare invece innaturale, in quanto sottrae alla storia del mondo classico la sua agonia. Le invasioni barbariche rappresentano d'altronde un continuum difficilmente sezionabile, diversamente dall'espansione araba che pone bruscamente termine alla dimensione universale dell'Impero ed all'unità culturale del Mediterraneo.

24 Per la particolare natura della religione ebraica, piuttosto fondata che etnica, e per il modo in cui essa ed il nascente cristianesimo trascendono la loro parziale appartenenza al mondo classico cf. BiANchi, La storia delle religioni, cit. (n. 12), p. 46 n.1. 
storia religiosa di popoli diversi ${ }^{25}$. Un ripensamento della natura delle fonti s'impone però più ancora come condizione di un migliore approfondimento, di una maggiore valorizzazione della loro intrinseca natura. Nella nostra tipologia abbiamo dato particolare rilievo alla distinzione tra fonti secondarie, che forniscono una rappresentazione dei fatti religiosi ad essi più 'prossima' (con le precisazioni che seguiranno), e fonti primarie, monumentali o scritte: tra quest'ultime andranno a loro volta distinte talune fonti ampie (tali da riportare un mito o un'azione rituale, così come dovevano essere conservati per ripetere l'azione religiosa) ed altre di natura più puntuale (iscrizioni dedicatorie, funerarie), non senza situazioni intermedie (leggi e regolamenti sacri, calendari). Le fonti primarie, specie quelle non ampie e soprattutto non mitiche, spesso non sembrano presentare allo studioso sufficienti elementi per un'interpretazione autonoma, cioè prevalentemente fondata sull'informazione che contengono, che peraltro in molti casi può essere raggiunta con un'analisi sistematica della documentazione primaria ${ }^{26}$. Ad un'insufficiente valorizzazione dell'informazione autonomamente presente in un complesso di fonti primarie, soprattutto se di carattere puntuale, si accompagna spesso la tendenza ad interpretare le fonti primarie come semplici occorrenze, banali conferme di schemi interpretativi ricavati senza problematizzazione critica dalle fonti secondarie prossime ai fatti: è il caso dell'interpretazione della religione greca, prevalentemente fondata - di fatto, implicitamente - sulle fonti letterarie ateniesi, senza una adeguata problematizzazione del rapporto tra queste e l'ingente documentazione epigrafica; ma è anche il caso del peso tuttora prevalente dell'interpretazione greco-romana e delle equivalenze stabilite dagli autori classici nella comprensione delle strutture religiose degli altri popoli del mondo classico (questa volta nel senso più ampio), anche di quelli dotati di un'ingente documentazione scritta.

La nostra esigenza di una più netta distinzione metodologica, che porti a cogliere separatamente (seppur in una comprensibile integrazione reciproca) le strutture proprie di singoli complessi documentari primari e gli schemi delle singole fonti secondarie, specie se non riflesse, parte anche da una rinnovata attenzione ai loro schemi concettuali. Il nostro atteggiamento implica il passaggio da un'impostazione, in cui sono parzialmente coestensivi i linguaggi

25 Prova ne è l'insuccesso di Historia religionum a cura di BLEEkER e Widengren, che voleva armonizzare la presentazione delle diverse religioni all'interno di un unico schema di riferimento.

26 Questo tipo di interpretazione abbiamo tentato, in forme diverse, in F. Mora, Prosopografia Isiaca. II. Prosopografia storica e statistica del culto isiaco, Leiden, 1990 (EPRO, 113) (in seguito: Prosopografia); ID., Die Griechiscben Tbeopboren Namen (im Bereich der griechiscben Inseln), in Pomoerium, 1 (1994), p. 15-35; Nomi teofori e polt-

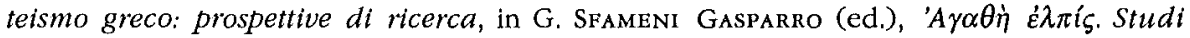
storico-religiosi in onore di Ugo Bianchi, Roma, 1995, p. 177-186; ID., Theopbore Namen als Urkunden lokaler Religion, in H.G. KIPPENBERG (ed.), Lokale Religionsgeschichte, DVRGTagungsband zur DVRG-Jabrestagung 1993 in Bremen, Marburg, 1995, p. 101-117. 
della fonte secondaria e dello studioso moderno, quest'ultimo distinto soprattutto da un maggior grado di riflessione e distanza, ad un'altra, che riconosce una diversa forma di riflessione e ricostruzione dei fatti nella fonte secondaria e nell'autore moderno ${ }^{27}$. L'informazione della fonte secondaria, secondo la nostra proposta, è costituita da materiale puntuale ed esteriore (' attendibile' per una critica positiva ed analogo a quello delle fonti primarie) assimilato - secondo modelli non sempre semplici e lineari - in schemi mentali, prevalentemente non espliciti, che forniscono (proprio in quanto impliciti) un'importante testimonianza sulla percezione dei fatti culturali o religiosi nella cultura prossima alla fonte secondaria.

La fonte secondaria può appartenere ad una cultura diversa da quella su cui riferisce: in questo caso si apre una problematica di grande interesse e solitamente trascurata, e cioè un'analisi delle testimonianze etnograficoreligiose volta non a studiare le civiltà testimoniate, ma a capire gli schemi che sorreggono la rappresentazione della civiltà ${ }^{28}$ e dei suoi elementi nella cultura dello storico (antico), anche alla luce della frequente differenziazione, in gradi diversi, tra cultura propria, affine, 'altra' o anche non-cultura. Le testimonianze antiche sugli dèi degli Sciti ci informano - con molti limiti sulla religione scitica, ma contemporaneamente l'insieme delle notizie antiche sugli dèi dei popoli limitrofi permette di delineare i meccanismi di assimilazione di divinità straniere e di rappresentazione delle proprie, in quanto intese come strutture universali utilizzabili quale strumento interpretativo. Gli dèi come forme del reale ${ }^{29}$ esistono (e sono correttamente analizzabili) soprattutto a questo livello ${ }^{30}$, in cui da una parte passano in secondo piano elementi secondari o meno attuali, dall'altra emergono - negativamente, per contrasto - quei tratti particolari, 'delicati ', che non danno luogo a generalizzazione; una simile analisi può aiutare a meglio cogliere le differenze tra forme divine apparentemente concorrenti. L'indagine sulle testimonianze incrociate ${ }^{31}$ permette d'altronde di approfondire in misura ancora maggiore la soggettiva percezione della religione, fino a tratteggiare, per singoli autori antichi, una tipologia della Religione in generale, dei suoi

27 L'acquisizione di un metalinguaggio storico-religioso, altro da quello ingenuo della cultura dello studioso o delle sue fonti, è quindi l'obiettivo della ricerca storicoreligiosa, non parte di una premessa metodologica, che in ultima analisi riposa su una implicita e riduttiva ontologia della religione (vuoi teologica, vuoi antiteologica).

28 Cf. F. MORA, Religione e religioni nelle Storie di Erodoto, Milano, 1986 (in seguito: Religione); ID., L'interpretazione delle collettività divine in Strabone $(X, 3)$ e la fenomenologia religiosa di Posidonio, in SMSR, 59 (1993), p. 7-19; ID., Il Pensiero storico-religioso antico. Autori greci e Roma: I, Dionigi d'Alicamasso (in seguito: Pensiero), Roma, 1995.

29 V. oltre, n. 49.

30 Cf. Mora, Religione, cit. (n. 28), p. 81-101 e Pensiero, cit. (n. 28), p. 7-42. V. anche oltre, p. 19.

31 Mora, Pensiero, cit. (n. 28); ID., Religionswissenschaftiches Denken in der Antike, in $Z R G G, 51$ (1999), p. 17-29; v. sopra, p. 15. 
elementi costitutivi e del rapporto tra la Religione in sé e le varianti ${ }^{32}$ costituite dalle singole religioni. ${ }^{33}$ Infine, sia che si tratti di fonti secondarie appartenenti ad una civiltà terza (rispetto alla nostra e a quella oggetto) sia che si tratti di fonti secondarie riflesse, è evidente il vantaggio rappresentato per un simile rinnovamento dell'analisi delle fonti secondarie da una diversa autonoma attenzione alle fonti primarie, quale quella più sopra accennata.

Delle religioni del 'mondo classico', nel senso più ampio del termine (tale cioè da comprendere anche le 'Alte culture del mondo antico ' e i 'Popoli limitrofi '), due, e cioè quella greca (d'epoca classica ed ellenistica) e quella romana (dalle guerre puniche a tutta l'epoca imperiale) si prestano in modo particolare alla ricerca moderna, perché rientrano particolarmente bene nella nostra categoria sub c), per la presenza di fonti monumentali e scritte, che comprendono anche testimonianze riflesse, ed ancor più per l'utile presenza di testimonianze incrociate. La religione ebraica, di cui abbiamo una buona conoscenza a partire dall'età monarchica ${ }^{34}$, ci è invece nota (fino all'età romana) solo attraverso fonti letterarie (con specifica esclusione di fonti monumentali di valore religioso), che comprendono rare testimonianze riflesse e quasi nessuna significativa fonte secondaria prodotta da membri di un'altra civiltà ${ }^{35}$ : l'interpretazione risulta quindi favorita e condizionata dalla successiva autocomprensione della duplice tradizione, giudaica e cristiana, che ad essa si riconduce. Alla nostra categoria d) appartiene anche la religione sumerica, nota attraverso fonti scritte e monumentali, di generi diversi, $m a$ in cui scarse risultano le testimonianze riflesse e le fonti secondarie esterne (scritte da membri di altre culture) ${ }^{36}$; il linguaggio religioso sumerico, fin nel suo senso più letterale della terminologia sacra e divina sumerica, condiziona poi significativamente la documentazione della religione assira, fino a far parlare di un'unica religione mesopotamica od assiro-babilonese ${ }^{37}$ (in quanto l'interpretatio potrebbe rispecchiare un'autentica assimilazione), ed in maniera ancor più curiosa quella relativa alla religione ittita, ben distinta da quella sumera, ma il cui pantbeon ci è noto solo nella traduzione 'scrittoria' sumera, che implica con ogni probabilità una significativa deformazione concettuale ${ }^{38}$.

32 Nella prospettiva che domina l'interpretazione antica, soprattutto greca, dei fatti religiosi.

33 Cf. Mora, Pensiero, cit. (n. 28), p. 383-412.

34 H. RINGgren, Israelitische Religion, Stuttgart, $1981^{2}$, tr. it. Milano, 1987, p. 81.

35 A. PENNA, La religione d'Israele, in Storia delle religioni, cit. (n. 12), III, p. 575-696, partic. p. 578,582 sq.

36 G.R. Castellino, La religione sumerica, in Storia delle religioni, cit. (n. 12), III, p. 155 , partic. 4.

37 L. CAGNI, La religione assiro-babilonese, in Storia delle religioni, cit. (n. 12), III, p. $57-125$, partic. p. $79-84$.

38 G. Furlani, La religione degli Hittiti, in Storia delle religioni, cit. (n. 12), III, p. 141182, partic. p. 148. 
Il netto prevalere, tra le fonti relative all'antico zoroastrismo, di un solo genere di testi, di tipo rituale, pur non precludendo la comprensione della religione persiana (che può fortunatamente avvalersi di fonti secondarie esterne), ne condiziona significativamente la ricostruzione; ${ }^{39}$ la presenza di insufficienti testimonianze riflesse rappresenta un grosso limite ${ }^{40}$ delle nostre conoscenze circa la religione della Siria antica, pur in presenza di fonti dirette e monumentali di una certa ampiezza; un problema di particolare rilevanza per la ricostruzione della religione fenicia è posto dalla marcata tendenza delle fonti secondarie esterne, relativamente importanti per la complessiva sistemazione dei fenomeni religiosi, ma caratterizzate da una percezione nettamente concorrenziale ed ostile, rispettivamente greca e romana, delle civiltà fenicia e punica ${ }^{41}$.

Ad un livello assai più debole di conoscenza si arriva nel caso di buona parte delle antiche religioni indoeuropee (Celti, Slavi, Balti, Germani), a noi note tramite fonti secondarie esterne, testimonianze archeologiche, l'interpretazione del sistema linguistico ed anche tradizioni conservate in fonti folkloristiche e leggendarie assai più tarde: l'uso della comparazione (in una prospettiva metodologica più marcatamente ricalcata su quella della comparazione linguistica ${ }^{42}$ ) come elemento essenziale della ricostruzione presuppone l'accettazione di un più modesto livello di conoscenza, realisticamente volto ad individuare le sole strutture profonde, senza pretendere di riconoscere, se non marginalmente, varianti e sviluppi specifici: il metodo comparativo come fattore principale se non esclusivo di individuazione ed interpretazione dei fatti a partire da frammenti troppo minuscoli porta dunque ad un appiattimento della comprensione idiografica dei fenomeni paragonabile alla deformazione dovuta alla concettualizzazione sumerica delle religioni mesopotamiche ed ittita.

39 G. GNol, La religione persiana, in Storia delle religioni, cit. (n. 12), III, p. 233-292 partic. p. 238.

40 G. Garbini, La religione della Siria antica, in Storia delle religioni, cit. (n. 12), III, p. 195-231, partic. p. 198.

41 S. Ribichini, Credenze e vita religiosa presso $i$ Fenici ed $i$ Cartaginesi, in Trattato, cit. (n. 2), III, Milano, 1992, p. 169-190 partic. 170.

42 Sia nella ricerca più tradizionale (ad es. G. Devoto, La religione degli indoeuropei, in Storia delle religioni, cit. (n. 12), III, p. 343-360; V. PISANI, Le religioni precristiane degli Slavi, dei Balti e dei Celti, ibid. p. 361-461; C.A. MAESTRELLI, La religione degli antichi Germani, ibid. p. 463-535; A. Prosdocimi, Le religioni dell'ttalia antica, ibid. p. 673-722; G. Devoto, Origini indoeuropee, Firenze, 1962; E. BENVENISTE, Le vocabulaire des institutions indo-européennes, I-II, Paris, 1969-1975, tr. it. Torino, 1976) sia nella ricostruzione assai più potentemente comparativa del DumÉzil (L'idéologie tripartie des Indo-Européens, Bruxelles 1958; Mythe et épopée, I-III, Paris, 1968-1973; Les dieux souveraines des IndoEuropéens, Paris, 1977; cf. J. RiEs, La méthode comparée en Histoire des religions selon Georges Dumézil et Mircea Eliade, in Notion, cit. (n. 1), p. 713-719 partic. p. 713-715). 
In altri casi a rendere significativamente più intelligibile una documentazione religiosa sono i suoi elementi di continuità con altre tradizioni religiose: è questo il caso delle religioni minoica e micenea, la cui ricostruzione, basata prevalentemente sulla documentazione archeologica, ma in misura rilevante anche su fonti scritte primarie, trae notevole giovamento dagli elementi di continuità con la religione greca ${ }^{43}$. Le diverse fasi dell'evoluzione religiosa degli Etruschi possono essere ricostruite individuando, sul piano linguistico, successive influenze esterne grazie alla conoscenza dei contesti religiosi (italico e greco) con cui gli Etruschi entrarono in contatto ${ }^{44}$; tale conoscenza favorisce in modo significativo una ricostruzione basata su fonti dirette, archeologiche ed epigrafiche (anche se la conoscenza degli elementi assorbiti dalla religione etrusca ben poco ci dice circa la loro originale rielaborazione $^{45}$ ): per cogliere nelle sue linee essenziali la particolarità di questo sistema religioso ${ }^{46}$ bisogna però ancora affidarsi alla percezione dell'alterità religiosa etrusca che ebbero le fonti secondarie esterne, greche e romane, senza sottovalutare il rischio di rielaborazioni tardive e non etrusche del modello etrusco ${ }^{47}$.

\section{Elementi di una tipologia delle religioni classiche}

Caratteristiche essenziali del politeismo ${ }^{48}$ sono l'organizzazione sistematica non geografica delle figure divine, la replicabilità in luoghi diversi di questa organizzazione ed il carattere astratto ed universale ${ }^{49}$ delle figure divine (che presuppongono forme di rapporto differenziate coi fedeli), spesso

43 B.C. Diztrich, Religione, culto e sacro nella civiltà cretese-micenea, in Trattato, cit. (n. 2), III, Milano, 1992, p. 69-90, partic. p. 86.

44 A. Maggiani, L'uomo e il sacro nei rituali e nella religione etrusca, in Trattato, cit. (n. 2), III, Milano, 1992, p. 191-209, partic. p. 197-199.

45 M. Eliade, Histoire, tr. it. cit. (n. 2), II, p. 130 sq.

46 In particolare l'orientamento cosmologico della teologia etrusca: MagGianr, art. cit. (n. 44), p. 201; U. BIANchI, Il cosmo come tempo e spazio qualificati nella religione degli Etruschi, in L. Bianchi (ed.), Gli Etruschi e Roma. Archeologia e storia religiosa. Atti del seminario di studi. Roma settembre-ottobre 1987, Roma, 1988, p. 101-113, partic. p. 107; Eliade, op. cit. (n. 2), p. 134.

47 Secondo il vecchio, ma non inattuale, monito di G.Q. GigLIoLI, La religione degli Etruscbi, in Storia delle religioni, cit. (n. 12), III, p. 537-672, partic. p. 544-552.

48 F. Mora, Per una tipologia del politeismo, in Notion, cit. (n. 1), p. 823-830 (in seguito: Politeismo).

49 Alludiamo alla definibilità degli dèi in termini concettuali, per cui W. Oтто, Die Götter Griecbenland, Bonn, 1929, tr. it. Firenze, 1941, 163 sq. vi colse le 'forme originarie della realtà ': per i limiti dell'Otto, quale ' teologo di una moderna religiosità pagana ', $c f$. H. CANCIK, Dionysos 1933, in R. FARBer, R. Schlesier (eds), Die Restauration der Götter, Würzburg, 1986, p. 105-123. 
accompagnate, quale elemento stabilizzatore, da una relativa laicizzazione ${ }^{50}$, sovente congiunta ad una tematica classificatoria: il politeismo greco rappresenta una forma assai marcata, compiuta di questa struttura religiosa, particolarmente protesa ad impostare in termini universalistici, per via di assimilazione, il rapporto con le altre forme religiose. Nei termini della moderna tecnologia informatica il politeismo greco si autocomprende come un superset, un linguaggio più potente in grado di racchiudere in sé, quali propri subsets, i politeismi degli altri popoli, (quasi) come un processore di nuova generazione deve poter eseguire quanto viene già eseguito dagli (ultimi) processori precedenti (con un certo grado di compatibilità retrograda).

La stessa percezione del politeismo come campo semantico particolarmente strutturato (e quindi oggetto di un'evoluzione lenta e delicata, perché ogni aggiunta non marginale provoca una ridefinizione complessiva del sistema), che sorregge la percezione moderna della mitologia classica quale eredità greco-romana ( $m a$ in realtà solo greca: v. oltre, p. 24) rispecchia in definitiva l'universalizzazione del pantbeon greco come insieme sovraordinato non solo ad ogni singolo pantheon locale greco, ma anche all'insieme dei sistemi religiosi barbarici: rispecchia quindi la pretesa che di ogni religione e di ogni singolo culto in ogni religione si possa e debba dare un'espressione possibilmente adeguata, ma quanto meno approssimata all'interno del sistema politeistico greco, l'imposizione del linguaggio mitologico greco come linguaggio religioso universale ${ }^{51}$, che implica il prevalere degli elementi concettuali se non classificatori su altre forme di organizzazione del mondo divino, in primis la semplice catalogazione geografica, come elencazione di culti percepiti come locali, non trasferibili. Probabilmente la ragione profonda di questa particolare evoluzione del politeismo greco risiede, almeno in parte, nella rapidità dell'espansione coloniale greca, che combinata con la tendenza anfizionica (a riunirsi in leghe locali) e la tendenza democratica a reinventare i rapporti sociali nell'esperienza coloniale ${ }^{52}$, facilita l'invenzione di una religione nazionale ${ }^{53}$, cioè condivisa da tutti coloro che parlano greco e facil-

50 Nel senso dell'assenza di un clero fortemente strutturato e depositario di un sapere religioso riservato quale premessa di una libera, carismatica reinterpretazione del patrimonio religioso tradizionale: $c f$. F. MORA, Storiografia greca e romana, in $D H A, 25$ (1999), p. 7-33, n. 33.

51 Tale cioè da permettere di esprimere non una, ma qualunque religione.

52 Sull'importanza della colonizzazione nel sorgere del miracolo greco $c f$. ad es. P. LÉvêQue, L'aventure grecque, Paris, 1964, tr. it. La civiltà greca, Torino, 1970, p. 188-214, partic. p. 212 .

53 Il criterio linguistico-nazionale ancora in uso per individuare gli oggetti dell'indagine storico-religiosa (del mondo antico) risente significativamente del punto di vista greco che potremmo indicare come il postulato della naturale coestensione di lingua e religione: chiunque parla una lingua appartiene a quella religione etnica, o ad una sua variante marginale locale, o da essa si è in qualche modo allontanato (per seguire un diverso stile di vita, ad es. orfico o pitagorico). Dove è forte una struttura templare, che porta all'adozione nel rapporto tra uomini e divinità di schemi interpretativi non indivi- 
mente riproducibile ${ }^{54}$, con minimi aggiustamenti, in ogni nuovo insediamento $^{55}$. Un qualche precedente del politeismo universale greco (quale forma cioè più generale di ogni singolo politeismo) può essere rappresentato dall'affermazione del linguaggio politeistico sumero ${ }^{56}$ (fin nel suo elemento più materiale, la forma scritta dei nomi divini) come linguaggio sovranazionale, utilizzato per esprimere le strutture divine di popoli e religioni diverse: una significativa differenza tra le due universalizzazioni di un sistema politeistico è nella maggiore concentrazione di quello greco, che, non affidato a specialisti e più libero dalle tendenze centrifughe della cultura templare, assume una forma essenziale, che si carica delle valenze e delle tensioni proprie di un campo semantico e può, parallelamente, venire interiorizzato come schema, adeguatamente sintetico, di percezione della realtà (v. sopra, p. 19).

A questa percezione universale, fortemente strutturata e piuttosto rigidamente immutabile del divino si accompagnano nella religione greca una marcata desacralizzazione della vita quotidiana, il depotenziamento e la sprofessionalizzazione dei sacerdozi cittadini e la concentrazione in santuari extraurbani, regionali delle forme più specializzate ed intense di rapporto col divino (comunicazione oracolare, culti di guarigione ed anche culti misterici),

duali, ma generici ed applicabili volta a volta a divinità diverse, l'ambito religiosamente omogeneo è per il fedele solo quello locale, anche se lo studioso moderno può riscontrare una forte affinità (tipologica e concorrenziale) tra diversi ambiti locali: l'unità della religione nazionale è in questi casi fondata sulle analogie tipologiche riconosciute dall'autore moderno (sulla scorta del criterio linguistico-religioso dei Greci antichi ed eventualmente dell'esistenza di strutture statuali unitarie) piuttosto che sulla soggettiva percezione dei fedeli.

54 Probabilmente all'iniziale debolezza dei singoli insediamenti si deve la maggiore accentuazione presso i Greci (rispetto ai Fenici, altrimenti contrassegnati da un'espansione coloniale per certi versi analoga) della tendenza anfizionica e comunque della maggiore tensione ad un'identità nazionale sovracittadina. Il maggiore particolarismo fenicio (che impedisce di parlare della religione fenicia come di un sistema religioso complessivamente omogeneo per il singolo fedele: $v$. sopra, $\mathbf{n}$. 53) può d'altronde forse dipendere dal permanere di una classe sacerdotale di specialisti, che rafforza la tendenza ad una 'organizzazione templare', cui abbiamo ricollegato una componente non politeistica, in chiave 'enoteista' (volta cioè all'affermazione della superiorità del proprio dio): cf. Mora, Politeismo, cit. (n. 48), p. 827-830.

55 Ibid., $824 \mathrm{sq}$.

56 Sull'influenza della mitologia orientale, ed anche sumera, sulla religione greca $c f$. U. BIANCHI, La religione greca, in Storia delle religioni, cit. (n. 12), III, p. 81-394, partic. p. 151; W. BURKERT, Die orientalisierende Epoche in der griechischen Religion und Literatur, Heidelberg, 1984. v. anche G. Komoroczy, The Separation of Sky and Earth. The Cycle of Kumarbi and the Myths of Cosmogony in Mesopotamia, in AAntHung, 21 (1973), p. 21-45; M.K. SCHRETTER, Alter Orient und Hellas. Fragen der Beeinflussung griechischen Gedankengutes aus altorientalistchen Quellen, dargestellt an den Göttern Nergal, Rescheph, Apollon, Innsbruck, 1974; J. O'Brien, W. MAJOR, In the Beginning. Creation Myths from Ancient Mesopotamia, Israel and Greece, Chicago, 1982. 
che richiedono una qualche forma di specializzazione sacerdotale ${ }^{57}$. Quale maggiore polarità emerge il contrasto tra una routine quotidiana relativamente desacralizzata ed il momento forte della comunicazione col divino, in un luogo eccezionale (il santuario) e/o con modalità eccezionali (dalla follia dionisiaca ai riti d'iniziazione), ma soprattutto in un momento reso speciale dalla sua dimensione di festa collettiva, prevalentemente di festa sovracittadina. Se esistono taluni canali per sollecitare una (difficile) comunicazione col divino, se taluni momenti ristabiliscono il contatto tra la massa dell'umanità ed il divino, la vita quotidiana rimane al riparo dalla presenza del divino e questo, profondamente caratterizzato dalla propria immutabilità, non incide, se non eccezionalmente, sulla storia umana ed in nessun modo viene caratterizzato dal suo rapporto con essa.

La religione greca risulta sotto questo aspetto diametralmente opposta a quella romana: all'assenza di reciproche interazioni tra le divinità nel mito si accompagna invece nella religione romana la caratterizzazione degli dèi in larga misura per il tramite dei loro interventi storici, delle loro ierofanie nell'agire storico-politico che ne fondano il culto o una festa, un tempio particolari; all'assenza di canali specializzati di rapporto col divino (per iniziativa umana), che viene compensata a partire da un certo momento con l'adozione di quelli greci (prima la consultazione degli oracoli greci, poi l'interesse per le iniziazioni nei santuari ellenici), si accompagna una costante preoccupazione di riconoscere, ma anche neutralizzare l'iniziativa divina volta a comunicare all'uomo (meglio: allo stato romano), per raggiungere (solo tardivamente) con la formalizzazione estrema dell'esperienza religiosa quell'autonomia dell'agire umano, quella libertà dagli dèi che sembra quasi il presupposto scontato della religione greca. Al carattere poco professionale del sacerdozio greco ed al suo forte orientamento sulla struttura del mondo divino (cui corrisponde prevalentemente uno ad uno) si contrappone in Roma l'accentramento delle funzioni sacerdotali in alcuni collegi di specialisti di ambiti diversi dell'interazione tra umano e divino, che, fortemente integrati nell'élite politica, rappresentano una struttura permanente di competenza e potere, in rapporto (tranne i flamines) con la totalità del divino e non con singole sue figure.

Se si considerano anche la totale assenza dalla religione romana del simbolismo sessuale così importante in quella greca, la forte preminenza del destino pubblico ${ }^{58}$ su quello privato a Roma ed il costante intervento rego-

57 Esito secondario di questa laicizzazione è l'esistenza di liberi professionisti del sacro, che coprono alcune lacune prodotte dalla riduzione dei sacerdozi civici ad una carica tra le altre: manteis che analizzano le offerte sacrificali prima della battaglia, venditori di oracoli, interpreti di sogni.

58 Diversamente affrontato nelle due religioni: Roma, pur importando in maniera superficiale il teatro greco, non riesce a far propria la ritualizzazione apotropaica della crisi possibile, operata ad Atene nella tragedia e nella commedia, perché è dominata dal 
latore dello stato romano nelle questioni religiose non si può non restare un po' stupiti dell'identificazione delle due religioni, operata a livello teorico ${ }^{59}$ tra il I sec. a.C. ed il I sec. d.C., sulla base di un numero relativamente ristretto di corrispondenze (il modello sacrificale ed il modello templare, d'architettura religiosa), che portano a maturazione, sistematizzandoli ed ampliandoli, gli elementi già gradualmente penetrati nella religione romana in virtù dei contatti avuti prima con le città della Magna Grecia, poi con gli stessi Greci della madrepatria e dell'Oriente ellenistico: decisiva in questo processo di assimilazione culturale ci sembra l'attrattiva di cui risulta portatrice la religione più complessa ed articolata sul piano della risposta ai bisogni individuali.

La forte polarità riscontrabile tra le due religioni, greca e romana ${ }^{60}$, permette di comprendere la strategia messa in atto da parte dell'apologetica cristiana, attaccando presso gli imperatori romani gli aspetti più specificamente greci della religione 'pagana', quasi a suggerire la presenza di altre, non meno significative possibili concordanze tra religione romana e cristiana. Punti di contatto possono essere del resto rintracciati, al di là delle profonde differenze (in primis quella fondamentale tra politeismo e monoteismo), tra religione romana ed israelitica: l'assenza del mito ${ }^{61}$, il ruolo dell'esperienza storica nella percezione del divino, la centralità religiosa del popolo piuttosto che dell'individuo, il rifiuto di culti sessualmente caratterizzati ${ }^{62}$ e i concetti affini di alleanza/pace con gli dèi ${ }^{63}$.

A rendere più difficile la conoscenza delle antiche religioni 'primitive' indoeuropee (Celti, Germani, Slavi, Balti) sono due diversi vizi di prospettiva nelle nostre fonti e nella metodologia cui dobbiamo ricorrere nella ricostruzione: a) la prospettiva ostile, distanziante delle fonti classiche e cristiane/

concetto di omen nefasto, cioè da una continuità positiva (e non da una presenza alternátiva) tra il simbolo e gli eventi futuri; cf. anche Mora, Storiografia, cit. (n. 50), n. 135.

59 Sulla religione 'greco-romana' quale costruzione teologica degli intellettuali greci d'epoca augustea, a partire da Dionigi cf. Mora, Pensiero, cit. (n. 28), p. 17-27, 413.

60 Presenza/assenza del mito e del simbolismo sessuale, diversità dell'organizzazione sacerdotale e delle forme di comunicazione tra uomo e divinità, diverso rapporto tra divinità e tempo storico, contrasto tra la centralità dell'individuo e dello stato nell'esperienza religiosa.

61 A. PENNA, La religione d'Israele, in Storia delle religioni, cit. (n. 12), III, p. 575-696, partic. p. 577; R. Bloch, La religione romana, in $\mathrm{H}$.-Ch. PuECH (ed.), Histoire des religions, Paris, 1970, tr. it Roma-Bari, 1976, III, p. 155-208, partic. 156 s.; BIANCHI, La storia delle religioni, cit. (n. 12), p. 116-120.

62 BIANCHI, La storia delle religioni, cit. (n. 12), p. 118.

63 M. Sordi, L'homo romanus; religione, diritto e sacro, in Trattato, cit. (n. 2), III, Milano, 1992, p. 285-307, partic. p. 287-291. 
islamiche, che tende a sottolineare gli elementi di alterita ${ }^{64}$ e $^{\text {barbarie }}{ }^{65}$; b) i meccanismi della comparazione, soprattutto di tipo duméziliano (peraltro in genere piuttosto convincente), che portano a privilegiare una comprensione di tipo greco degli elementi politeistici delle singole religioni, quali occorrenze di una più generale struttura religiosa indoeuropea, assai coesa.

Alla prospettiva greca di un politeismo quale religione nazionale, consistentemente omogenea nelle diverse repliche locali esperite come pienamente equivalenti dai singoli fedeli, risulta nettamente contrapposta, nel più ampio ambito del politeismo, una prospettiva assai diversa, che limita significativamente la componente classificatoria del politeismo greco, ed è dominata dall'importanza, nell'esperienza religiosa individuale e collettiva, della struttura templare, il cui forte sacerdozio, proteso ad affermare in chiave enoteista la superiorità del proprio dio, sviluppa schemi interpretativi non individuali, ma generici ed applicati di volta in volta a divinità diverse, ed in particolare la figura del dio creatore. A questa prospettiva si avvicina anche la concentrazione di molti tratti in una figura prevalente, localmente determinata, che caratterizza il particolare sviluppo del politeismo fenicio, quasi per atrofizzazione della componente classificatoria, probabilmente presente nella religione ugaritica ${ }^{66}$.

La divergenza dal modello greco di politeismo è ancor più evidente nel caso della religione egizia, in cui manca un sistema classificatorio ad un tempo locale e nazionale e le caratteristiche intrinseche non permettono di definire una singola divinità, ma rinviano (lo studioso moderno) a gruppi di divinità affini venerate in luoghi diversi, così che si può ricorrere quale principium individuationis solo alla combinazione di un criterio geografico e di un altro basato su tratti funzionali e più ancora teriomorfici. La mancata distinzione dipartimentale di funzioni e caratteristiche diverse determina poi un'anomala tendenza a combinare liberamente figure divine per realizzarne altre, in netto contrasto con la fondamentale distinzione greca tra figure complesse e singoli loro tratti: mentre l'unica operazione possibile in Grecia è la sottolineatura di un particolare tratto di una figura complessa, in Egitto la regola è la combinazione di tratti e figure diverse. A questa svalutazione delle figure divine socialmente riconosciute tramite il contrassegno del nome si accompagna d'altronde la tendenza di ogni divinità ad assumere su scala

64 Assenza di una cultura templare quale elemento essenziale della civiltà per le fonti classiche, caratterizzazione sfavorevole degli elementi politeistici/prepoliteistici estranei al monoteismo nelle fonti cristiane/islamiche.

65 Insistendo particolarmente sugli aspetti inumani del culto, quali i sacrifici umani e l'uso di teschi sacri. Nel caso dei Celti risultano fortemente deformate dall'inserimento in una tipologia greca, volutamente universalistica (per inglobare nella stessa antropologia greci e barbari) anche le notizie relative ai Druidi, che risalgono in buona misura a Posidonio, così come quelle circa la divinazione (funzionali alla sua tesi dell'ampia diffusione del sacrificio divinatorio presso i barbari).

66

MorA, Politeismo, cit. (n. 48), p. 828. 
locale una gamma ampia, quasi completa di attributi e funzioni, secondo lo schema ' enoteistico-templare' già ricordato parlando della religione fenicia.

Un'articolata tipologia delle religioni classiche richiede poi un'analisi sistematica di altre grandezze fondamentali, oltre alla struttura del mondo divino, ed in particolare: sul piano della spiegazione sacralizzante della realtà, la questione dell'origine del mondo e della realtà (cosmogonia ${ }^{67}$ ) e più in generale il rapporto tra la realtà presente e quella originaria/definitiva (protologia / escatologia ${ }^{68}$ ), il problema dei valori morali, della colpa individuale $^{69}$ e della presenza del male ${ }^{70}$, quale deficienza ontologica (teodicea ${ }^{71}$ ), la percezione della vita umana e del suo compimento, quale trascendimento della realtà esperibile (soteriologia ${ }^{72}$ ); sul piano della concreta organizzazione sacrale dell'esistenza umana, le forme di reclutamento ed organizzazione sacerdotale e le altre forme qualificate d'esperienza religiosa, i modi della sacralizzazione del potere ${ }^{73}$ (regalità sacra), l'organizzazione sacrale del tempo e dello spazio ${ }^{74}$ (calendario ${ }^{75}$ ed architettura religiosa), le forme di

67 Cf. U. BIANCHI, Teogonie e cosmogonie, Roma, 1960.

$68 C f$. ad es. U. BIAnchi, Prometeo, Orfeo, Adamo. Tematiche religiose sul destino, il male, la salvezza, Roma, 1976;. ID., Protologia, I. Nozioni generali: tipologia storico-religiosa, in A. Di Berardino, Dizionario Patristico e di Antichità Cristiane, II, Torino, 1984, p. 2929-2934; U. BIANCHI (ed.), La tradizione dell'enkrateia. Motivazioni ontologiche $e$ protologiche. Atti del Colloquio Internazionale. Milano, 20-23 aprile 1982, Roma, 1985; M.V. CERutTr (ed.), Apocalittica e gnosticismo (Tavola rotonda, Roma 18-19 giugno 1993), Roma, 1995.

69 U. BIANchi, Péché originel et péché antécédent, in RHR, 170 (1966), p. 117-126.

70 U. BIANCHI, Il dualismo religioso. Saggio storico ed etnologico, Roma, $1982^{2}$ [1958].

71 U. Branchi, Per la storia della teologia dei Greci: la 'Teogonia' di Esiodo, in La coscienza religiosa del letterato pagano, Genova, 1987, p. 9-26; ID., Théologie et théorie du mal aux premiers siècles de l'ère nouvelle, in Le Muséon, 100 (1987), p. 1-11; ID., Il male nel dualismo gnostico, in Liberaci dal male, Bologna, 1989, p. 199-208; ID., Teologia e teodicea: tra espressione e testimonianza, in A. BABOLIN (ed.), Testimonianza religiosa $e$ forme espressive, I, Perugia, 1989, p. 307-317.

72 U. Bianchi, M.J. Vermaseren (eds), La soteriologia dei culti orientali nell'Impero romano. Atti del colloquio internazionale, Roma 24-28 settembre 1979, Leiden, 1981; U. BIANCHI, Categorie e figure del salvifico nella storia delle religioni, in Religioni $e$ Società, 3 (1988), p. 9-13; ID., La soteriologia del cristianesimo. Ricerche storico-comparative, Roma, 1992.

73 D. Sabbatucci, Il mito, il rito, la storia, Roma, 1978; B. Gladigow, Die sakralen Funktionen der Liktoren, in ANRW, I 2 (1972), p. 295-364.

74 BIANCHI, Il cosmo..., cit. (n. 46); J.P. VERnANt, Hestia-Hermès: sur l'expression religieuse de l'espace et du mouvement chez les Grecs, in Mythe et pensée chez les Grecs, Paris, 1985, p. 155-201; B. Gladigow, Audi Juppiter, Audite Fines, in O. BeHrends, L. Capogrossi Colognesi (eds), Die Römiscbe Feldmeßkunst, Göttingen, 1992, p. 172-189.

75 U. BIANCHI, Ore, stagioni e tempi nella teoria politeistica del divino, in Religioni $e$ Società, 2 (1987), p. 7-11; D. MikAlson, The Sacred and Civil Calendar of the Athenian Year, Princeton, 1975; J. RÜPKE, Kalender und Öffentlichkeit, Berlin, 1995 (RGVV, 40). 
comunicazione col divino ed indagine del futuro (divinazione ${ }^{76}$ ), i modi della sacralizzazione degli elementi della vita quotidiana (soprattutto alimentazione ed abito), delle tappe principali ${ }^{77}$ dell'esistenza individuale (nascita, iniziazione, matrimonio ${ }^{78}$, morte $^{79}$ ) e dei momenti critici della vita collettivà (guerra ${ }^{80}$, epidemia, carestia); su quello infine di un'indagine sociologica dei fatti religiosi, la specificità dell'esperienza religiosa di singole categorie di fedeli (donne, militari, schiavi, ma anche ad es. naviganti) ${ }^{81}$.

\section{Paradigmi come strumento di un'analisi diacronica delle religioni classiche}

H. Küng ha convincentemente proposto la trasposizione del concetto di paradigma dall'ambito delle scienze naturali a quello della religione per distinguere forme successive di aggregazione degli elementi in stadi diversi di una tradizione, marcata da fattori sia di continuità sia di discontinuità. Così si può meglio comprendere l'interna organizzazione di un sistema religioso, studiandone l'evoluzione diacronica non come semplice aggiunta o perdita di elementi, ma piuttosto come trasformazione dei rapporti degli elementi rimasti tra loro e con quelli sopraggiunti. L'individuazione di diversi stadi puo in qualche misura risultare arbitraria selezione all'interno del continuum sto-

76 R. Flacelik̀re, Devins et oracles grecs, Paris, 1961; R. ВLосн, Les prodiges dans l'antiquité classique, Grèce, Étrurie et Rome, Paris 1963; A. CAquOT, M. LEIBovici, La divination, Paris, 1968; W. Burkert, Itinerant Diviners and Magicians. A Neglected Element in Cultural Contacts, in R. HÄGG (ed.), The Greek Renaissance of the eighth century B.C.: tradition and innovation, Stockholm, 1983, p. 115-119; R. BLOCH, La divination dans l'antiquité, Paris, 1984

77 U. BIANCHI, Transition Rites. Cosmic, social and individual order - I riti di passaggio. Ordine cosmico, sociale, individuale, Roma, 1986.

78 I. JENkIns, Is there Life after Marriage? A Study of the Abduction Motif in Vase Paintings of the Atbenian Wedding Ceremony, in BICS, 30 (1983), p. 137-145.

79 V. LANTERNARI, Orgia sessuale e riti di recupero nel culto dei morti, in SMSR, 24-25 (1953-54), p. 163-288; D.C. Kurtz, J. BoArdman, Greek Burial Customs, Ithaca-New York, 1971; R. Garland, The Greek Way of Death, Ithaca-London, 1985.

80 A. BReLICH, Guerre, agoni e culti nella Grecia arcaica, Bonn, 1961; W.K. PRITchetT, The Greek State at War, III: Religion, Berkeley, 1979; R. LonIs, Guerre et religion à l'époque classique. Recherches sur les rites, les dieux, l'idéologie de la victoire, Paris, 1979; F. GRAF, Women, War and Warlike Divinities, in ZPE, 55 (1984), p. 245-254; J. RüPKE, Domi militiae. Die religiöse Konstruktion des Kriegs in Rom, Stuttgart, 1990; H. WIssmanN (ed.), Krieg und Religion, Würzburg, 1994; H. von StIETEncron, J. Rüpke (eds), Töten im Krieg, Freiburg, 1995.

81 F.I. ZeItuIn, Cultic Models of the Female. Rites of Dionysius and Demeter, in Arethusa, 15 (1982), p. 129-157; Mora, Prosopografia, cit. (n. 26), II, p. 2-29 (cf. M. Malaise, Prosopographie et diffusion isiaques, in Kernos, 6 [1993], partic. p. 369-371); N. BоËLsJANSSEN, La vie religieuse des matrones dans la Rome archaique, Rome, 1993; C. SCIBONA GIUfFré, Culti femminili e identità della donna nella religione greca, Roma (in corso di pubblicazione); F. BÖMER, Untersucbungen über die Religion der Sklaven in Griecbenland und Rom, Wiesbaden, 1960 sq.; D. WACHSMUTH, POMPIMOS O DALMON, diss. Berlin, 1967. 
rico, risponde però al procedimento per salti discreti che caratterizza l'evoluzione dei fenomeni socio-culturali che abbiano espressione istituzionale, in cui un lungo processo di erosione sbocca, con svolta epocale, in una nuova e diversa aggregazione quale espressione di riferimento. Analizzando il fenomeno in termini sociologici, ci si può aspettare di osservare, al momento della svolta epocale, il prevalere come nuova espressione culturale dominante di una struttura di pensiero, che era precedentemente espressione di una variante minore, sub-culturale della stessa civiltà/religione e, spesso, il ricomparire del precedente paradigma fondamentale quale variante secondaria, sub-culturale.

Per l'Ebraismo Küng ha individuato come prima approssimazione, sulla base del resto di una scansione temporale già comune nella ricerca scientifica, la successione dei paradigmi tribale, monarchico, ierocratico (postesilico), rabbinico-sinagogale (medievale, conservatosi fino al XVIII secolo) e assimilazionistico della modernità (almeno fino all'Olocausto). Dal punto di vista storico-religioso lo schema utilizzato (e che ancor troppo risente di un'impostazione teologica) appare semplificare in maniera eccessiva, soprattutto perché non si pone il problema della dialettica tra paradigma prevalente e linee di tendenza alternative o, in altri termini, tra cultura mainstream e sub-culture: si deve ad es. sicuramente lamentare la scarsa attenzione nella macroperiodizzazione agli sviluppi escatologici prima, apocalittici poi del giudaismo postesilico ${ }^{82}$, all'insorgere di diversi stili di vita religiosa (in particolare esseni e farisei) all'interno della religione ebraica ${ }^{83}$, all'evoluzione in senso intellettuale della religione ebraica, quale religione della parola sacra ${ }^{84}$,

82 Küng non solo trascura gli aspetti escatologici del giudaismo immediatamente postesilico (per cui $c f$. M. Eliade, Storia delle credenze e delle idee religiose, II, Firenze, 1980, p. 249-256), ma non coglie (Das Judentum, tr. it. Milano, 1995, p. 146-148) l'alterità del modello apocalittico rispetto al paradigma teocratico, per quanto riguarda sia la forte apertura ad influenze straniere, ellenistiche (ELIADE, ibid., p. 275), sia il contrasto profondo tra l'apertura universalistica dell'escatologia ebraica e la chiusura 'nomocratica ' operata, intorno alla Tôrâh ed al suo commento, per salvare l'identità di Israele quale ' struttura etnica consacrata dalla sua stessa origine' (Eliade, ibid., p. 277).

83 Questi dimostrano la soggettiva percezione della semplice appartenenza etnica ad Israele quale insufficiente garanzia di identità religiosa, in quanto l'appartenenza etnica, condizione necessaria ma non sufficiente, deve essere riguadagnata a livello di ortoprassi mediante una radicale distinzione dei comportamenti.

84 Questa evoluzione, che trova espressione anche nella canonizzazione della Scrittura e della liturgia, accanto e poi (dopo la distruzione del secondo tempio) al posto dell'azione rituale, serve anche a ribadire il tradizionale rifiuto delle analogie antropologiche tra la propria prassi religiosa e quella dei vicini: il rifiuto del sacrificio fuori da Gerusalemme e poi, distrutto il tempio quale luogo di identità religiosa, del sacrificio tout court, nasconde anche la paura che il sacrificio possa rappresentare un elemento di omologazione alla prassi religiosa ellenistica. Una svolta epocale nell'antropologia religiosa (il passaggio da un paradigma incentrato sul sacrificio alimentare ad un altro incentrato prevalentemente sulla parola) ha quindi come significativa concausa la radicale tendenza ebraica alla propria alterità, disomologazione religiosa dal contesto circostante. 
che garantisce la continuità del giudaismo dal ritorno dall'esilio babilonese fino a tutta la diaspora.

Qui di seguito vogliamo provare a tratteggiare un'analoga analisi, quale successione diacronica di paradigmi, delle religioni greca e romana. Il principale criterio di scansione diacronica della religione greca ci sembra il ruolo del mito e della sistemazione razionale, filosofica nell'esperienza religiosa: ad una prima fase mitopoietica (e di codificazione del mito nella tradizione epica, omerica ed in quella, assai diversa, esiodea) succede (nell'epoca assiale) una fase di riflessione sistematica sul mito, alla luce di principi e riflessioni filosofiche, largamente improntate ad una diversa rappresentazione del divino, concepito come unico ed amorfo, e comunque a preoccupazioni d'ordine morale (soprattutto sul male e sulla causalità); la revisione critica del mito politeista alla luce di una diversa tendenza ' monoteista ${ }^{85}$ sui generis porta d'altronde alla radicale subordinazione della religione tradizionale alla speculazione filosofica, con il risultato che i veri detentori del sapere su questa vita (cioè della prospettiva che questa vita orienta ed in qualche misura sacralizza) sono in epoca ellenistica i filosofi ${ }^{86}$ delle diverse scuole, mentre il mito viene degradato da messaggio portatore di una rottura di livello ad elemento da riassorbire, mediante l'interpretazione allegorica, in un sapere diversamente prodotto. L'ulteriore evoluzione di questo paradigma centrale della religione greca è data dall'evoluzione in senso misteriosofico dei misteri ${ }^{87}$, gli unici culti oggetto, nella terza fase della nostra classificazione, di un più accentuato interesse: sotto l'influenza del dualismo platonico (che a sua volta media anche analoghe tematiche orfiche) il tema della caduta delle anime viene a dar nuovo impulso, in prospettiva anticosmica, a quell'elemento iniziatico-soteriologico, prima relativamente marginale rispetto ad una religiosità dominata dal modello olimpico ${ }^{88}$.

Tra la seconda e terza fase (rispettivamente riflessione critica sul mito, adozione della filosofia come chiave interpretativa della realtà) si pone anche la più marcata cesura sociologica della religione greca, e cioè il passaggio dalla civiltà greca a quella ellenistica; fuori dalla madrepatria l'elemento greco non rappresenta più la totalità organica di una popolazione, ma solo il suo strato dominante (mai superiore al 10\%), Questa situazione ripete, in termini più radicali, l'esperienza coloniale in Magna Grecia e porta alla compresenza

85 Sulle tendenze monoteiste nelle religioni classiche $c f$. F. Mora, Arnobio e $i$ culti di mistero. Analisi storico-religiosa del V libro dell'Adversus Nationes, Roma, 1994, p. 98 n. 156; C. Rowe, One and Many in Greek Religion, in Eranos-Jb., 45 (1976), p. 37-67.

86 Il passaggio è favorito dalla ' laicità ' della religione greca per cui v. sopra, n. 50.

87 U. Bianchi, Initiation, mystères, gnose, in C.J. BLeEker, Initiation. Contributions to the Theme of the Study-Conference of the I.A.H.R., Strasbourg, September 17th to 22nd 1964, Leiden, 1965 (Numen, suppl. 10), p. 154-171.

88 U. BIANCHI, Saggezza olimpica e mistica eleusina nell'inno omerico a Demetra, in SMSR, 35 (1964), p. 161-193. 
di due livelli dell'esperienza religiosa, la religione greca e le superstizioni ${ }^{89}$ locali; a questa distinzione di livelli, uno vero, l'altro falso, si lega forse il sorgere della teologia politica, che considera parte degli usi religiosi falsi, ma utili al governo delle masse ${ }^{90}$.

Se il discorso sugli dèi si trasforma gradualmente da fondamentale messaggio religioso a semplice bene di cultura, facilmente esportabile ${ }^{91}$ ma già avvicinabile, come nell'esperienza rinascimentale, alla fiction, il discorso falso, o addirittura da ridurre ad un elemento linguistico, ad un significante in cerca di significati (con una banalizzazione che peraltro impone l'uso del linguaggio religioso greco), costante rimane nella religione greca l'importanza del modello alimentare sacrificale (con cui si confrontano infatti anche gli stili di vita alternativi, in primis il bios pitagorico): in epoca imperiale la decadenza riguarderà piuttosto il sistema oracolare centralizzato in alcuni santuari specifici, anche sotto l'influsso del diverso sistema romano di prendere gli auspici e della concorrenza degli specialisti etruschi della comunicazione divinatoria.

Nella religione romana ci sembra possibile individuare una prima fase arcaica, in cui il discorso sugli dèi e le pratiche religiose informano ancora effettivamente l'esperienza dello stato romano, al cui interno forse si dovrà ancora distinguere un primo, più antico strato indoeuropeo (che determina soprattutto la struttura del mondo divino) da un altro più recente, etrusco, responsabile della particolare attenzione romana alla comunicazione divina (auguri, aruspici, le dottrine relative agli omina) ${ }^{92}$ e forse più in generale della sacralizzazione delle strutture spaziali e politiche (pomerium, littori) ${ }^{93}$. Decisamente specifico ${ }^{94}$ è nella religione romana l'accentramento, forse protorepubblicano, del sacerdozio in collegi con ampie competenze, che porta alla formazione di una élite politico-religiosa molto forte e conservatrice; questa, se risulta ' laica' per la particolare commistione di funzioni religiose e politiche, non lo è però nel senso greco, di una funzione sacerdotale generica ed indifferenziata, si caratterizza al contrario per un potere fondato sul proprio sapere religioso.

89 Il tema della superstizione $(\delta \varepsilon \iota \sigma ı \delta \alpha \iota \mu$ oví $\alpha)$ è introdotto da Teofrasto, ma sviluppato sistematicamente da Antipatro di Tarso verso la metà del II sec. a.C.

90 Sulla distanza tra élites e masse e la gestione di una religione non più condivisa $c f$. anche Mora, Pensiero, cit. (n. 28), p. 409.

91 Veicolo del mito greco, ad es, in ambito etrusco e romano, sono vasi ed altri utensili istoriati con rappresentazioni mitografiche, probabilmente accettate nel loro valore più superficiale, almeno nel mondo etrusco, che assimila gli eroi, ma non gli dèi greci: Maggiani, art. cit. (n. 44), p. 197.

92 Gf. anche Sord, art. cit. (n. 63), p. 292.

93 Cf. FEST. 285; SORDI, art. cit. (n. 63), p. 303.

94 Come mostra la sorpresa greca per tale anomala organizzazione del sacerdozio romano: Mora, Pensiero, cit. (n. 28), p. 236-251, 391-397. 
Nel III secolo successivo si colloca una rapida, per certi versi un po' superficiale $^{95}$, ma al tempo stesso piuttosto radicale ellenizzazione (in chiave ellenistica) della cultura romana, che porta in particolare alla scoperta della categoria greca di storia, e quindi alla reinvenzione annalistica del proprio passato $^{96}$ : la storia romana è quindi piena di mito storicizzato anche perché è stata scritta nella prospettiva ellenistica che storicizza o riduce a fiction il mito. Della visione ellenistica, più sopra delineata, della religione quella romana riprende altri elementi rilevanti: la relativizzazione dei concetti religiosi e la loro subordinazione ad altri elementi costitutivi della realtà (con una preferenza però per le strutture politiche piuttosto che per le riflessioni filosofiche), con una marcata contrazione della potenziale 'rottura di livello', anche per quanto riguarda temi specificamente romani come la pax deorum; l'approdo (nel I secolo) alla gestione da parte di un'élite illuminata, profondamente estranea al patrimonio religioso romano, di una religione per le masse quale strumento di integrazione sociale ${ }^{97}$; una nuova insistenza su personalità eccezionali, quasi versioni minori dei re ellenistici in qualche modo sovraumani, ed una nuova concezione del rapporto privilegiato tra capi politici e le loro personali divinità protettrici; l'apertura a culti greci ed orientali, quale risposta ad una domanda religiosa diversamente impostata, nei termini più individualistici di una richiesta di salvezza o comunque di attenzione al destino personale.

Il cambiamento più radicale della religione romana è però rappresentato dall'adozione del culto imperiale e dal ruolo centrale da esso acquisito: la religione romana viene ripensata in una prospettiva universale, cioè nón più come religione di una città, ma come fondamentale tessuto culturale dell'Impero, elemento di coesione tra Roma e le province, strumento quindi di una limitata, ma efficace assimilazione delle aree conquistate; in questa riscrittura della religione romana (in parte sorretta nella sua prospettiva universalizzante dal carattere universale del mito greco come discorso sugli dèi quali forme universali del divino) l'insistenza sul culto imperiale ${ }^{98}$ come forma di lealismo politico verso Roma e di coesione dell'Impero si carica sia delle attese di

95 Mora, Storiografia, cit. (n. 50), p. 11-12, con n. 32-34.

96 Ibid., p. 7-12.

97 Lo svuotamento della religione tradizionale risulta a Roma sotto questo aspetto assai più radicale che in Grecia, dove la reinterpretazione filosofico-allegorica del patrimonio mitico tradizionale conserva, almeno al livello dei significanti, un elemento di continuità tra le masse e le élites che le gestiscono. Per questo non appare insensato il tentativo cristiano, cui abbiamo già accennato (v. sopra, p. 25) di incrinare la religione romana, proponendo alle élites il monoteismo cristiano quale religione filosofica, teologia naturale, contrapposta sia alla teologia civile (rivolta alle masse, ora integrabili in prospettiva diversa, cristiana) sia a quella favolosa (patrimonio greco, ma non romano): cf. MoRA, Amobio, cit. (n. 85), p. 27.

98 Su cui v. R. TuRCAN, Culto imperiale e sacralizzazione del potere nell'impero romano, in Trattato, cit. (n. 2), III, Milano, 1992, p. 309-335. 
evergetismo politico-religioso, proprie soprattutto dell'Oriente ellenistico ${ }^{99}$, sia di elementi di sacralizzazione dello stato e dell'imperatore, che, nelle loro forme più marcate (ma anche maggiormente estranee al sistema possono ricollegarsi, sia pure in scala minore, alla funzione di centro vivente e sacrale dello stato proprio del faraone nella religione egizia. L'aspetto più interessante della religione d'epoca imperiale è però il complesso e dinamico equilibrio di elementi diversi all'interno (italici, greci, ' barbari ', orientali) e (prevalentemente) all'esterno (giudaici e cristiani) di un unico sistema religioso, che risente fortemente del diverso indirizzo dato dai singoli imperatori ${ }^{100}$.

Università di Messina

Fabio MORA

Dipartimento di Studi Tardi Antichi

Facoltà di Lettere

Via dei Verdi

I - 98122 MEssinA

99 Mora, Prosopografia, cit. (n. 26), II, p. 72.

100 Per un'analisi di questa problematica rimandiamo al nostro Zur kaiserlicben Steuerung der römiscben Religion, in corso di pubblicazione. 